\title{
MODELING AND PARAMETER IDENTIFICATION OF VIBRATIONS OF A DOUBLE TORSION PENDULUM WITH FRICTION
}

\author{
Ernest CZERWIŃSKI*, Paweł OLEJNIK*, Jan AWREJCEWICZ*
}

*Department of Automation, Biomechanics and Mechatronics, Lodz University of Technology, ul. Stefanowskiego 1/15, 90-924 Łódź, Poland

ernest.czerwinski@dokt.p.lodz.pl, pawel.olejnik@p.lodz.pl, jan.awrejcewicz@p.lodz.pl

received 23 August 2015, revised 9 December 2015, accepted 10 December 2015

\begin{abstract}
The purpose of this paper is to investigate a double torsion pendulum with planar frictional contact. The single torsion pendulum with one-degree-of-freedom is an angular equivalent of the linear harmonic oscillator. The second degree of freedom has been obtained by adding a free body to the inverted single torsion pendulum. The free body's angular displacement is caused by frictional forces appearing in the interface (contact zone) between the free body and the pendulum column's head kinematically excited at its base by a mechanism with torsion spiral spring. An experimental station has been set up and run to find most unknown parameters of the pendulum from the time series of state variables taken as inputs to the Nelder-Mead method of identification. The obtained results proved significant usability of the identification method in the case of numerical simulation of the pendulum's dynamical model. It has not been satisfactorily proved in the case of time characteristics coming from a real system that exhibits also some unrecognized physical effects.
\end{abstract}

Keywords: Friction, Planar Contact, Numerical Modeling, Double Torsion Pendulum, Parameter Identification, Nonlinear Vibrations, Kinematic Excitation, Kinematic Forcing Mechanism, Nelder-Mead Method

\section{INTRODUCTION}

The friction in nature exists in the form of external friction occurring at the interface of two bodies. Two kinds are distinguished: a static friction between the two mutually not moving bodies; a kinetic friction between the two mutually moving bodies: friction sliding, wherein the speed of the two bodies in their mutual contact points are different; friction bearings, wherein the resistance movement occurs in turning one body after another. The friction may have a structural form that is related to a dissipation of energy released in the contact surface of mutually fixed parts. Finally, the friction may be internal being observed in solids and fluids in the form of mechanical energy loss (Awrejcewicz and Olejnik, 2005).

The first full friction model was presented by Charles Coulomb, who showed that the static friction is not constant, and pointed to the variability of kinetic friction. It is often used in engineering practice. The concept of friction can incorporate the friction effects such as: a viscous friction increasing linearly while the speed increases; the Stribeck effect observed when a friction force decreases at a low speed, which is called the Stribeck speed; the friction dependent on angular position of the contact surface in rotational motion (Awrejcewicz and Olejnik, 2007).

Steady states of a nonlinear discrete three-degree-of-freedom system containing a torsional damper are investigated in Skup (2002). The system under consideration is harmonically excited. The analysis takes into account structural friction and linear viscous friction of a ring floating in a plunger filled with a high density silicon oil. The influence of external loading amplitude, unit pressures, linear viscous damping, geometric parameters and amplitude-frequency characteristics is analyzed. The equations of motion of the examined power transmission system are solved by a slowly-varying parameter method and digital simulation.

The described system is related to our work too, in a few key points, i.e. by regarding to the influence of friction in the twodegrees-of-freedom system as well as in the forced oscillation of the torsion pendulum caused by harmonic excitation.

In Bassan et al. (2013) an analysis of a simple torsion pendulum's motion is presented. Usual basic dynamical model is investigated in the context of some unexpected features found in experimental data. Comparison with observed values yields estimates for the misalignment angles and other parameters of the model. The authors developed a more flexible model for the torsion pendulum. The basic feature of that is to consider a rigid body suspended to the fibre at an arbitrary point, therefore, not necessarily associated to any particular symmetry of the body. Despite maximum experimental accuracy, some misalignment can occur when the fibre is fastened to the test mass. A detailed mechanical model of the torsion pendulum with geometrical imperfections can explain two unexpected features, ie.: the modulation of the torsion signal at the natural frequency of the swinging motion and the splitting of the swinging resonance.

Analysis of a torsion pendulum is also conducted in some experimental works. For instance, the dynamic process from perioddoubling bifurcations to chaos is observed in Miao et al. (2014) by changing the driving period of a modified Pohl's torsion pendulum that formally exhibits periodical dynamics. A data acquisition system with a CCD camera connected to a computer and corresponding image processing software is designed to exhibit the dynamics of the modified pendulum by recording the oscillating angle of the copper rotating wheel. As a result, abundant chaotic sequence diagrams and phase diagrams can be clearly seen in real time. 
The described system is related to our work by a similar form of kinematic forcing. With regard to the method of tunable parameters of periodic forcing and the free body being in a frictional contact with the second body, our experimental model is capable to exhibit any irregular behavior, including various ways of parameter dependent bifurcation diagrams and chaotic motion.

An eight-degrees-of-freedom Lagrangian model that provides a suitable account for the motion of the double torsion pendulum is described in De Marchi et al. (2013). The model fully describes its free dynamics and its response to external disturbances, and can accurately predict the torsion, swinging pendulum and bouncing resonances. The number and location of resonance peaks are correctly predicted and are used for a first validation of the model with preliminary data. There is possible to extend the Lagrangian formalism, via the Rayleigh dissipation function, to account for frictional forces. Dissipative effects are taken into account in order to predict, via the fluctuation-dissipation theorem, the fundamental limits of sensitivity of the system.

The described system is related to our work in two key points: (a) parameter identification during validation of the investigated pendulum's model; (b) estimation of frictional forces that act in the contact interface described in Section 2.

Physical systems such as an inverted pendulum driven by a spiral spring, an unbalanced Euler elastica with a travelling mass, a heavy body with a parabolic section and an Ising ferromagnet are very different. In Coullet et al. (2009) a nonlinear oscillator close to its supercritical bifurcation that oscillates with a period inversely proportional to its angular amplitude is presented. The authors performed experiments with a Holweck-Lejay-like pendulum which was used to measure the gravity field during the twentieth century. Main conclusions prove, that the spiral spring, parabolic heavy body and Holweck-Lejay oscillators belong to the same class of universality as the Ising ferromagnet close to their symmetry-breaking bifurcation. The Larmor's law was confirmed experimentally with a good accuracy.

The described system is related to our work in the key point: kinematic forcing of the investigated double torsion pendulum is made with the use of a spiral spring. Thanks to that, the dynamics may be investigated using similar methods taken into consideration in Coullet et al. (2009).

The real application of torsional dynamics can be found in Liu et al. (2014) showing modeling and analysis of a drilling system discretized into several components with lumped inertia properties and with the inertia elements interconnected with axial and torsional springs. The work extends the model of one coupling of two mutually rotating bodies onto a set of multiple couplings modelling the dynamics of a drill string.

The described system is related to our work only in its particular connection, but it brings a real application that could be investigated using elementary couplings with a frictional interface and a torsional spiral spring.

In other fields of science like in textile metrology or biology the double torsion pendulum is found as the basic model for the derivation of principles governing description of dynamics of complex hybrid systems.

In Michalak and Krucińska (2004), the influence of chemical treatment on bending and torsional rigidity of flax and hemp fibers was studied. The double pendulum's mathematical model is useful for the determination of fibre bending rigidity, and the torsional pendulum is helpful in a subsequent determination of fibre torsional rigidity in investigation of bast fibre rigidities.

The nonlinear dynamics of DNA relevant to the transcription process in terms of a chain of coupled pendulums was described in Cadoni et al. (2013). The authors provided a simple model for a nonlinear double chain showing some features which are quite interesting both in the frame of nonlinear dynamics for discrete systems and for applications, in particular, to DNA torsional dynamics.

\section{A PHYSICAL MODEL OF THE DOUBLE TORSION PENDULUM WITH FRICTION}

Fig. 1 shows a three-dimensional simplified visualization of the double torsion pendulum without any excitation mechanism. It consists of main and auxiliary parts such as screws and pins. The construction is inverted as compared with the general models of torsion pendulums so that on the pendulum column's head it is possible to freely place a next body. Rotational displacement of the free body depends on the pendulum column's dynamics and the friction in the interface of the two contacting bodies. The lower part of the sleeve is connected to the movable cam with the torsion spiral spring imitated by a handmade bar spring. A dynamic kinematic forcing of the free end of the spring attached to the slider causes the pendulum's torsion about an angle. The construction provides an angular contact bearing, allowing the rotation of the column with minimizing the adverse effects of friction occurring in that part of the construction (see Fig. 3).

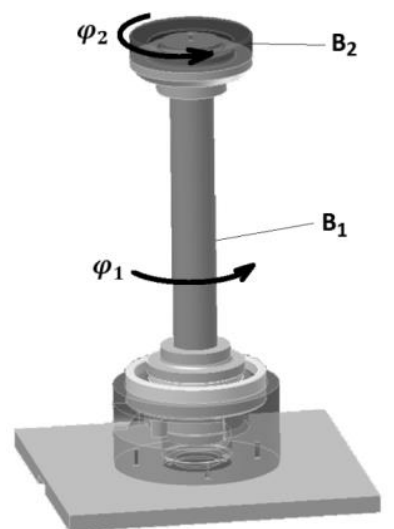

Fig. 1. The double torsion pendulum's $C A D$ in Inventor with marked generalized coordinates $\varphi_{1}$ and $\varphi_{2}$

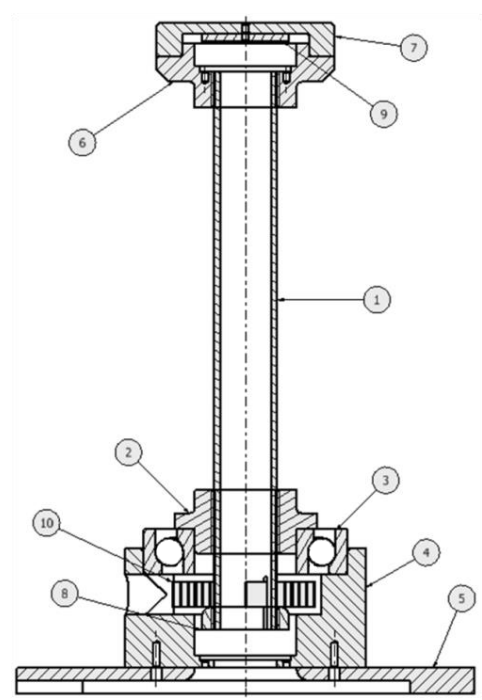

Fig. 2. Scheme of the double torsion pendulum 
In Fig. 2 it can be seen a cross-section taken along the vertical axis of the pendulum with the numbered particular elements. The construction is placed on the plate (5) on which the pendulum's base (4) is mounted. The base is designed so that one can attach the lower measuring circuit board of circular shape, bearing (3) and the torsion spiral spring (10). Angular contact bearing that is used in the project transmits the rotation to the lower mounting ring (2), which is screwed onto the sleeve (1). Rotational movement is generated by the spiral spring mounted between the body and the sleeve. At the end of the sleeve there is concealed a plastic cap (8), while the other end of the sleeve is engaged with an upper mounting ring ( 6 - head of the pendulum's column), wherein the second measuring circuit board with the angular motion sensor is placed. A free body (7) with a screwed plastic cap (9) containing two small circular magnets is placed on the upper surface of the mounting ring. Two rotating magnets attached to the free body create a contactless measurement by means of the Hall-effect sensor of magnetic field. Therefore, any angular relative displacement of the bodies can be measured.

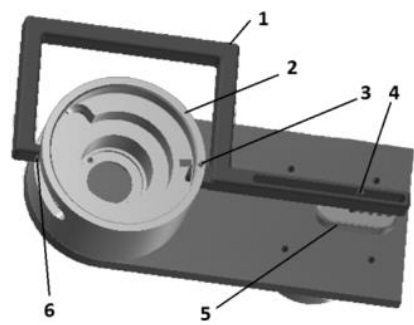

Fig. 3. A mechanism generating the dynamic kinematic sinusoidal forcing (see $f_{e}(t)$ given by equation (3b)) of one end of the torsion spiral spring

The design provides some space for installation of the two mentioned circuit boards with the Hall-effect sensors, which measure angles of rotation of the torsion pendulum's column formed by elements (1,2 and 6) and the internal sleeve bearing (3), as well as an angle of rotation of the free body (7).

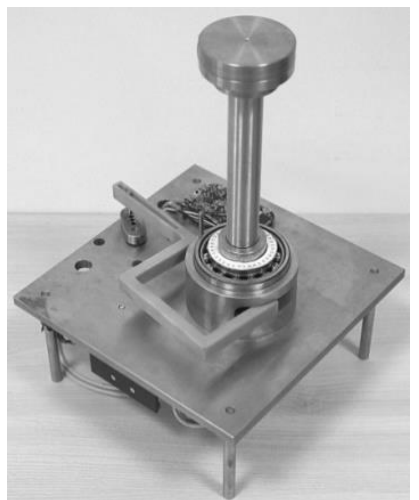

Fig. 4. View of the experimental stand with electronics and a stepper motor mounted below the base plate

The torsion pendulum's kinematic forcing mechanism visible in Fig. 3 consists of a few mechanical parts (1-5) and a stepper motor attached to the cam (5). The cam (5) which is put on the stepper motor's shaft enables movement of the arm (1) via an intermediate pin (4). The point of rotation of the arm is the axis of the fastening pin (3) in the pendulum's base $(2-$ it is the same base marked by 4 in Fig. 2). At the end of the arm there is a roller
(6) used to attach one end of the spiral spring. The second end is attached to the sleeve (1) visible in Fig. 2.

Finally, the described design has been physically realized, and a view of the experimental stand for investigation of dynamics of the two-degrees-of-freedom mechanical system with friction is shown in Fig. 4.

\section{ELECTRONIC SYSTEM FOR MEASUREMENT OF THE VECTOR OF GENERALIZED COORDINATES}

The vector of real measurement data used for the identification purposes has been subject to an acquisition by means of a measurement system shown in Fig. 5.

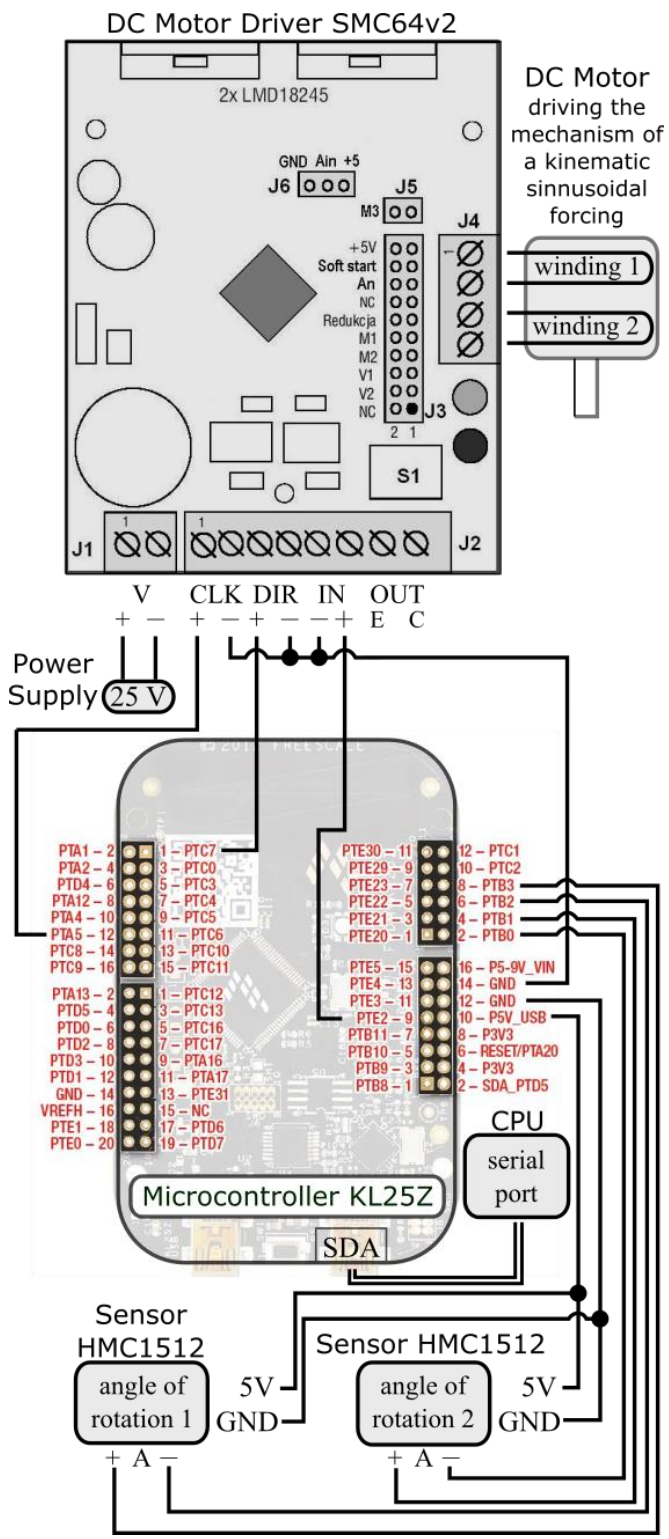

Fig. 5. The measurement and motor driving electronic system

The measurement and motor driving system of the torsion pendulum cosists of the following elements: two angular position sensors HMC1512 embedded in a dedicated electronic circuit based on the LM358 amplifier; Freescale microcontroller FRDMKL25Z for data acquisition and control; the DC stepper motor 
driver SMC64v2 controlled by the microcontroller; the 2-phase DC stepper motor 57BYG081 (55Ncm, 5V, 1A, $1.8^{\circ}$ - basic step); a computer (CPU in Fig. 5) for data reading in a serial connection with the SDA port of the microcontroller. After reading, the two series of data corresponding to the angles of rotation were presented in the Terminal in a column form and after a single experiment saved in a text file for further presentation on the graphs (see Fig. 11-13).

\section{MATHEMATICAL MODEL OF THE DOUBLE TORSION PENDULUM WITH FRICTION}

\subsection{Introduction}

The investigated double torsion pendulum with friction is an extension of a single torsion pendulum. The construction has been inverted, compared to the general models of torsion pendulum so that on the pendulum column's head there is possible to place a next body, of which free motion dynamics will depend on the base pendulum's dynamics and the friction forces acting in an interface of the two contacting bodies. This implies that the resulting frictional coupling with the two movable bodies establishes the two-degrees-of-freedom mechanical system with friction.

In Fig. 1 we have introduced two variables $\varphi_{1}$ and $\varphi_{2}$ that constitute the vector of generalized coordinates:

$$
q=\left[\begin{array}{l}
\varphi_{1} \\
\varphi_{2}
\end{array}\right]
$$

where $\varphi_{1}$ [rad] is the angular displacement of the pendulum's column relative to its base, $\varphi_{2}$ [rad] - the angular displacement of the free body relative to the column.

The zero position of the column is determined by the neutral position of the kinematically forced end of the spiral spring, which is attached at the center of the hollow bore's opening in the base. The column can rotate clockwise and counter-clockwise, contrary to the range indicated by the opening angle of the hollow bore.

\subsection{Derivation of the double torsion pendulum's equations of motion using the Lagrange method}

Kinetic energy of the double torsion pendulum is given by:

$T=\frac{1}{2} B_{1} \dot{\varphi}_{1}^{2}+\frac{1}{2} B_{2}\left(\dot{\varphi}_{1}+\dot{\varphi}_{2}\right)^{2}$,

where $B_{1}\left[\mathrm{~kg} \cdot \mathrm{m}^{2}\right]$ is the mass moment of inertia of the sleeve with two rings constituting the pendulum's column, $B_{2}\left[\mathrm{~kg} \cdot \mathrm{m}^{2}\right]$ - the mass moment of inertia of the free body.

The potential energy is given by:

$V=\frac{1}{2} k\left(f_{e}(t)-\varphi_{1}\right)^{2}$,

where $k[\mathrm{~N} \cdot \mathrm{m} / \mathrm{rad}]$ is the torsion spring's stiffness, $f_{e}(t)$ - function of the kinematic sinusoidal forcing (external excitation) of one end of the torsion spring given by the formula:

$f_{e}(t)=A \sin (\omega t)$,

where $\omega[\mathrm{rad} / \mathrm{s}]$ is the angular frequency of kinematic forcing, $A$ [rad] - amplitude of the kinematic forcing.

Vector $Q$ of generalized forces can be written as follows
$Q=\left[\begin{array}{c}\tau_{1} \\ \tau_{2}\end{array}\right]=\left[\begin{array}{c}-M_{L}+M_{T} \\ -M_{T}\end{array}\right]$

where $M_{L}[\mathrm{~N} \cdot \mathrm{m}]$ is the frictional resistance torque of the bearing, in which the first pendulum's body (the column) is mounted, $M_{T}$ $[\mathrm{N} \cdot \mathrm{m}]$ - the frictional resistance torque between both pendulum's bodies of inertia $B_{1}$ and $B_{2}$.

We assume, that the frictional resistance torque of the bearing depends on unknown viscous friction and the Coulomb friction expressed by the maximum static friction torque $M_{1}$ acting in the contact zone Michalak and Krucińska (2004). Moreover, the discontinuity introduced by Coulomb model of dry friction is smoothened by the smoothing function arctg approximating function $\operatorname{sgn}\left(\dot{\varphi}_{1}\right)$ of sign of the angular velocity $\dot{\varphi}_{1}$ of the column

$M_{L}=c_{1} \dot{\varphi}_{1}+M_{1} \frac{2}{\pi} \operatorname{arctg}\left(\varepsilon_{1} \dot{\varphi}_{1}\right)$,

where $c_{1}[\mathrm{~N} \cdot \mathrm{m} \cdot \mathrm{s} / \mathrm{rad}]$ is the coefficient of viscous friction in bearing, $M_{1}[\mathrm{~N} \cdot \mathrm{m}]$ - maximum torque of the static friction of the bearing in the contact zone, $\varepsilon_{1}[\mathrm{~s}]$ - a parameter determining accuracy of smoothing of the static friction torque's Coulomb term acting in the bearing's contact zone. The larger the value of $\varepsilon_{1}$ is the closer the arctan function approximates the frictional effects caused by Coulomb friction.

For further use in the text, the term "contact zone" will denote a contact zone in the frictional coupling between the pendulum's bodies of inertia $B_{1}$ and $B_{2}$.

One takes into account more frictional effects existing in the contact zone between both pendulum's bodies. The frictional resistance torque between bodies of inertia $B_{1}$ and $B_{2}$ depends on the viscous friction $T_{v} \dot{\varphi}_{2}$, smoothened relation for Coulomb dry friction $T_{s 1} \frac{2}{\pi} \operatorname{arctg}\left(\varepsilon_{2} \dot{\varphi}_{2}\right)$, the free body's angular position dependent friction $T_{s 2}\left(1-s g n\left|\dot{\varphi}_{2}\right|\right)$ and the Stribeck effect that is characterized by a Stribeck curve $T_{s t}\left(1-e^{-T_{0}\left|\dot{\varphi}_{2}\right|}\right) \operatorname{sgn}\left(\dot{\varphi}_{2}\right)$. Therefore, concatenating the possible frictional effects we obtain:

$M_{T}=T_{v} \dot{\varphi}_{2}+T_{s 1} \frac{2}{\pi} \operatorname{arctg}\left(\varepsilon_{2} \dot{\varphi}_{2}\right)$

$+T_{s 2}\left(1-\operatorname{sgn}\left|\dot{\varphi}_{2}\right|\right)+T_{s t}\left(1-e^{-T_{0}\left|\dot{\varphi}_{2}\right|}\right) \operatorname{sgn}\left(\dot{\varphi}_{2}\right)$,

where $T_{v}[\mathrm{~N} \cdot \mathrm{m} \cdot \mathrm{s} / \mathrm{rad}]$ is the viscous friction coefficient in contact zone, $T_{s 1}[\mathrm{~N} \cdot \mathrm{m}]$ - the maximum static friction torque in the contact zone, $T_{s 2}[\mathrm{~N} \cdot \mathrm{m}]$ - the maximum static friction torque, $T_{s t}[\mathrm{~N} \cdot \mathrm{m}]-$ friction coefficient associated with the exponential curve appearing due to the Stribeck effect, $T_{0}[\mathrm{~s}]$ - the parameter of exponential curve, $\varepsilon_{2}$ [s] - a parameter of static friction torque in the analyzed contact zone.

Expressing the kinetic and potential energies in generalized coordinates, a Lagrange function $L$ is defined as the difference of the kinetic energy $T$ and the potential energy $V$ :

$L=T-V$,

which after substituting equations (2) and (3) leads to the formula

$L=\frac{1}{2} B_{1} \dot{\varphi}_{1}^{2}+\frac{1}{2} B_{2}\left(\dot{\varphi}_{1}+\dot{\varphi}_{2}\right)^{2}-\frac{1}{2} k\left(f_{e}(t)-\varphi_{1}\right)^{2}$.

Then, for the function $L$, the Lagrange equation is used

$\frac{d}{d t}\left(\frac{\partial L}{\partial \dot{q}_{l}}\right)-\frac{\partial L}{\partial q_{i}}=Q$,

where $Q$ - vector of generalized forces, $q_{i}-i$-th generalized coordinate.

The Lagrange equation for the coordinate $\varphi_{1}$ is given by: 
$\frac{d}{d t}\left(\frac{\partial L}{\partial \dot{\varphi}_{1}}\right)-\frac{\partial L}{\partial \varphi_{1}}=\tau_{1}$

Considering only the left-hand side of equation (10) and equation (8), one obtains:

$\frac{d}{d t}\left(\frac{\partial L}{\partial \dot{\varphi}_{1}}\right)-\frac{\partial L}{\partial \varphi_{1}}=B_{1} \ddot{\varphi}_{1}+B_{2}\left(\ddot{\varphi}_{1}+\ddot{\varphi}_{2}\right)-k\left(f_{e}(t)-\varphi_{1}\right)$.

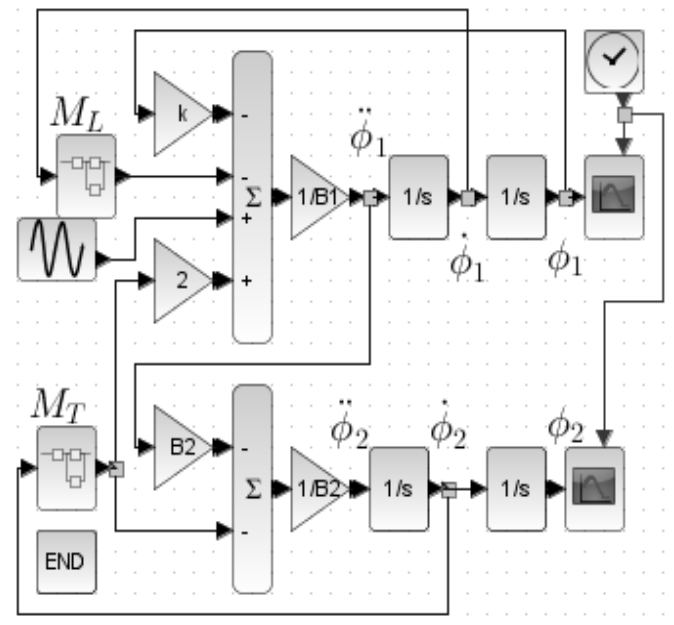

Fig. 6. Complete simulation diagram of the double torsion pendulum.

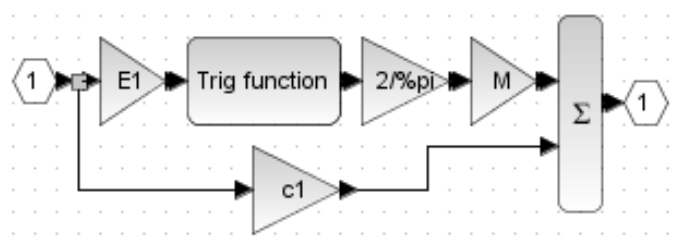

Fig. 7. Simulation diagram of a subsystem modelling the equation of the frictional resistance torque $M_{L}$

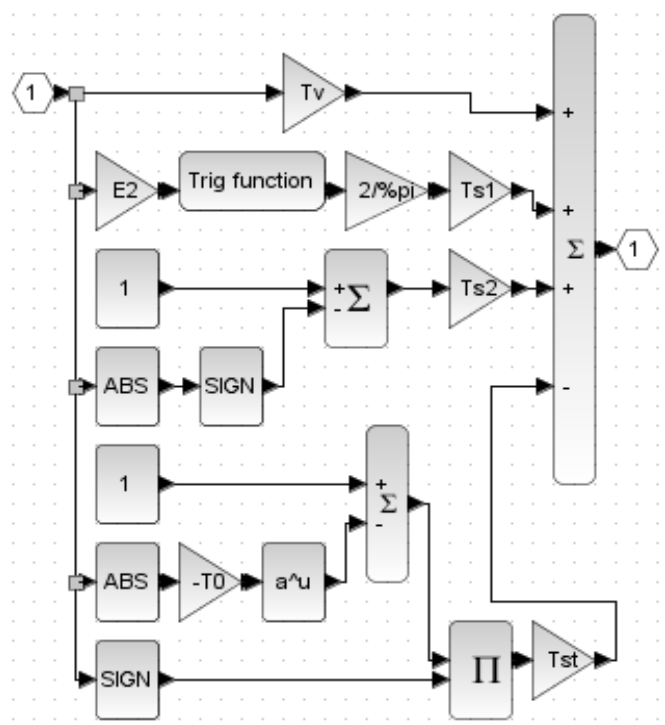

Fig. 8. Simulation diagram of a subsystem modelling the equation of the frictional resistance torque $M_{T}$ form:

The Lagrange equation for the coordinate $\varphi_{2}$ is given in the

$\frac{d}{d t}\left(\frac{\partial L}{\partial \dot{\varphi_{2}}}\right)-\frac{\partial L}{\partial \varphi_{2}}=\tau_{2}$.
Combining the left-hand side of equation (12) and equation (8), one gets:

$\frac{d}{d t}\left(\frac{\partial L}{\partial \dot{\varphi}_{2}}\right)-\frac{\partial L}{\partial \varphi_{2}}=B_{2}\left(\ddot{\varphi}_{1}+\ddot{\varphi}_{2}\right)$.

Substituting the generalized forces $\tau_{1}$ and $\tau_{2}$, which are defined in (4) into the equations (10) and (12), and taking into account the formulas (11) and (13), the two ordinary differential equations of second order describing the dynamics of the double inverted torsion pendulum with the dynamic kinematic forcing are found:

$B_{1} \ddot{\varphi}_{1}+B_{2}\left(\ddot{\varphi}_{1}+\ddot{\varphi}_{2}\right)-k\left(f_{e}(t)-\varphi_{1}\right)=-M_{L}+M_{T}$

and:

$B_{2}\left(\ddot{\varphi}_{1}+\ddot{\varphi}_{2}\right)=-M_{T}$.

Finally, the double torsion pendulum with a plane frictional coupling is represented by a two-degrees-of-freedom dynamical system and described by the system of two second-order ordinary differential equations:

$\left\{\begin{aligned} B_{1} \ddot{\varphi}_{1}-k\left(f_{e}(t)-\varphi_{1}\right) & =-M_{L}+2 M_{T}, \\ B_{2}\left(\ddot{\varphi}_{1}+\ddot{\varphi}_{2}\right) & =-M_{T} .\end{aligned}\right.$

Equations (16) were implemented in Scilab to build the simulation diagram of the analyzed physical model visible in Fig. 6-8.

\section{PARAMETER IDENTIFICATION OF THE REAL OBJECT}

Prior to the process of identification, the objective function was assumed as the arithmetic average of squares of the differences between the measured and estimated angles in the following form

$y=\frac{\frac{1}{N} \sum_{i=1}^{N}\left(\varphi_{1}-\widehat{\varphi}_{1}\right)^{2}+\frac{1}{N} \sum_{i=1}^{N}\left(\varphi_{2}-\widehat{\varphi}_{2}\right)^{2}}{2}$,

where $\varphi_{1}(t), \varphi_{2}(t)$ are the actual measurements of angles, $\hat{\varphi}_{1}(t), \hat{\varphi}_{2}(t)$ - the estimates of angles, $N$ - number of samples in the series. The objective function (17) was implemented in Scilab (see Listing 1 in Appendix).

To identify the unknown parameters of the pendulum the Nelder-Mead simplex method for finding a local minimum of a function of several variables has been used. It allows to determine the local minimum without using the derivatives, so it can be applied when the function is not differentiable at a point. In the numerical analysis we used an identification algorithm available in the Scilab (see Listing 1). Exemplary references extending the problem of finding of unknown parameters with the use of the Nelder-Mead simplex method can be found in Luersen and Le Richie (2004).

In the identification process there are assumed some known parameters: $\omega, \varepsilon_{1}, \varepsilon_{2}$, while the unknowns are as follows: $k, A, B_{1}, B_{2}, c_{1}, M_{1}, T_{v}, T_{s 1}, T_{s 2}, T_{s t}, T_{0}$.

\subsection{An identification based on the numerical solution - example}

Before the target identification of parameters of the real torsion pendulum will be made, a test of the assumed method in an exemplary simulation has to be carried out with the use of a nu- 
merically computed solution. The identification procedure was initiated with all values of parameters, i.e.: $\omega=0.5, \varepsilon_{1}=$ $1000, \varepsilon_{2}=1000, \mathrm{~B}_{1}=20, \mathrm{~B}_{2}=1, \mathrm{k}=100, \mathrm{~A}=\frac{\pi}{6}, \mathrm{c}_{1}=$ $10, \mathrm{M}_{1}=3, \mathrm{~T}_{\mathrm{v}}=1, \mathrm{~T}_{\mathrm{s} 1}=2, \mathrm{~T}_{\mathrm{s} 2}=2, \mathrm{~T}_{\mathrm{st}}=0.5, \mathrm{~T}_{0}=10$. As a result, the trajectories of state variables visible in Fig. 9 and 10 were obtained.

Figs. 9a and 9b show a time histories of system variables in a response of the pendulum to the sinusoidal function of the kinematic forcing $f_{a}(t)$. According to the specificity of the externally forced two-degrees-of-freedom dynamical system, the angular velocities and accelerations are difficult to predict within a time period of applied forcing. This irregularity is caused by the effects of a rolling friction present in the bottom bearing and the inertia of the free body acting reversely on the column at the stick-slip frictional contact interface of both bodies of the pendulum. In Fig. 9 we can see a temporal slippings as well as close to zero relative velocities of the free body against the column. Also in this case, the angular velocity and acceleration of both bodies are difficult to predict. It is caused by the frictional effects occurring at the frictional contact interface of both pendulum bodies of inertias $B_{1}$ and $B_{2}$.

a)

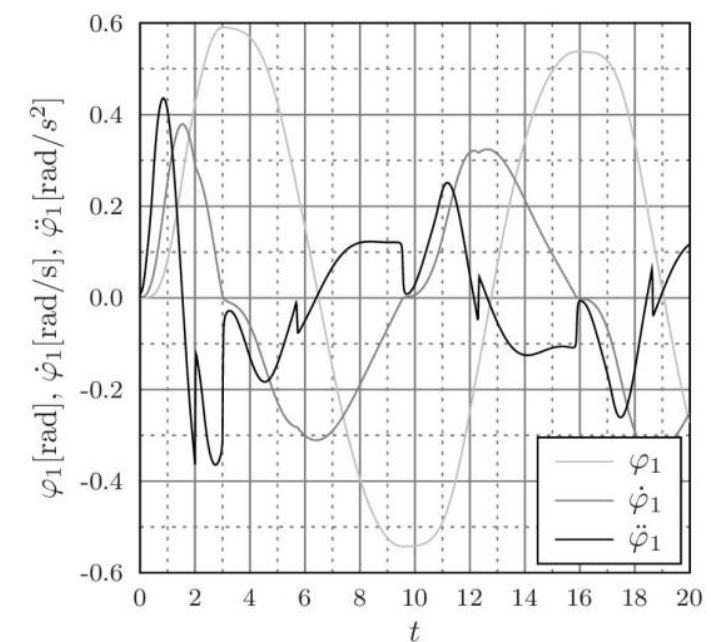

b)

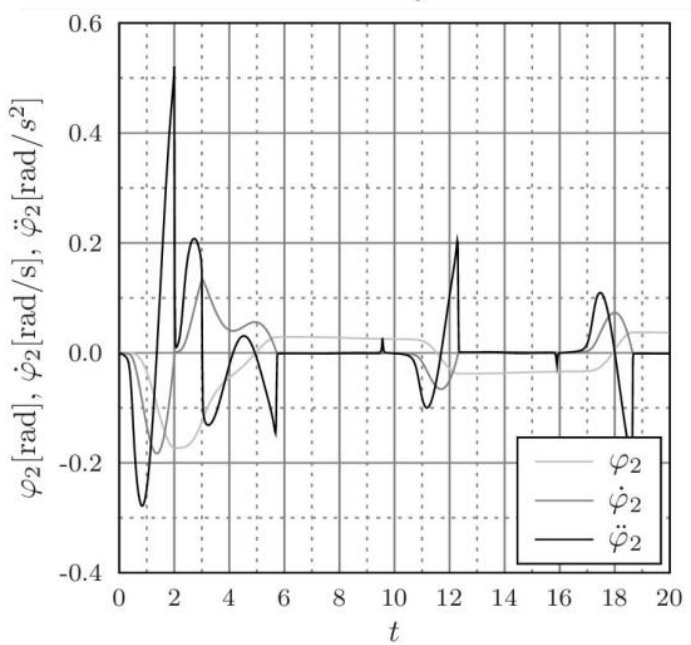

Fig. 9. Time histories of the angular displacements $\left[\varphi_{1}, \varphi_{2}\right]$, velocities $\left[\dot{\varphi}_{1}, \dot{\varphi}_{2}\right]$ and accelerations $\left[\ddot{\varphi}_{1}, \ddot{\varphi}_{2}\right]$ of the column and the free body

The set of parameters which were described as unknowns was slightly modified in relation to the simulation, i.e.: $B_{1}=22$, $B_{2}=11, \quad k=10, \quad A=\pi / 4, c_{1}=5, \quad M_{1}=2, \quad T_{v}=0.1$, $T_{s 1}=3, T_{s 2}=1, T_{s t}=0.2, T_{0}=1000$.
It is seen, that after a few steps of the identification procedure of parameters of the pendulum, the time trajectories are significantly different (see Fig. 10a and 10b). However, after several thousands of iterations, there was obtained better set of parameters. The angular velocity and acceleration are difficult to predict by unrecognized effects of friction occurring in contact zone of the pendulum's bodies.

a)

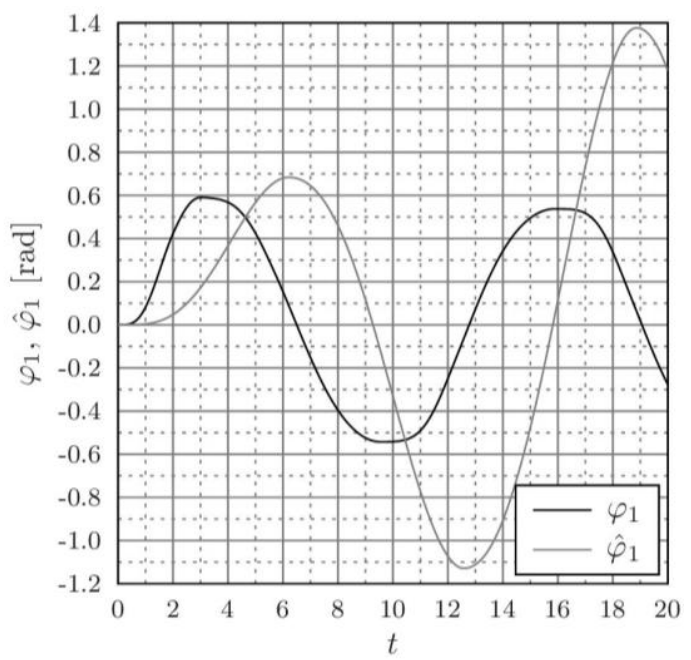

b)

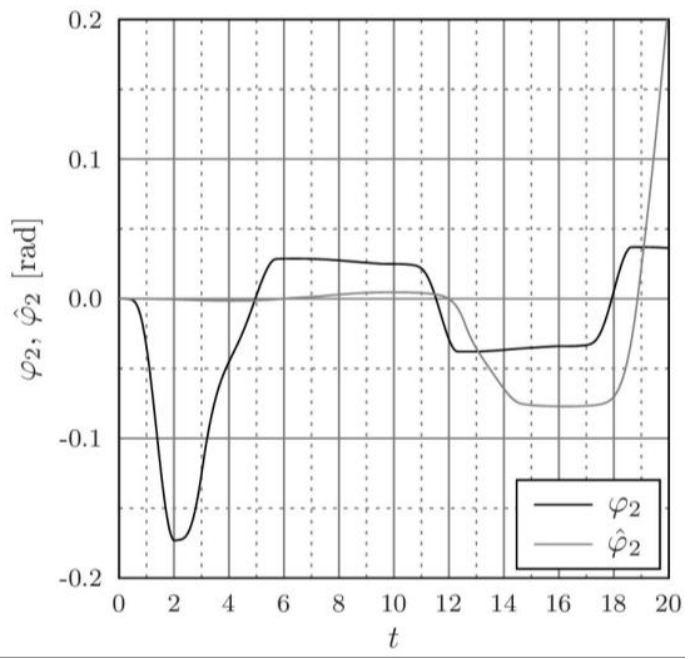

Fig. 10. Time histories of the actual $\left[\varphi_{1}, \varphi_{2}\right]$ and partially estimated $\left[\hat{\varphi}_{1}, \hat{\varphi}_{2}\right]$ dynamical variables of the pendulum before the final identification parameters are found. In the presented view, the model's parameters are not finally identified (the procedure of identification is still in progress)

It has guaranteed almost perfectly identified model of the inverted double torsion pendulum with friction and a dynamic kinematic forcing (see Fig. 11a and 11b).

\subsection{The target identification based on the data acquired from measurement}

In previous section a numerical solution of the pendulum's model was taken into consideration leading to the successful identification. Correctness of our methodology has been proved.

Now, beginning with the identification of the measurement series, three parameters were assumed as known. Frequency of the dynamic kinematic forcing $\omega=2 \pi / T=2 \pi / 0.48=13.09$ is assumed. A parameter of static friction torque in the slip zone of 
the bearing and the static friction torque in the analyzed frictional contact of the pendulum's bodies are as follows: $\varepsilon_{1}=$ $1000, \varepsilon_{2}=1000$, respectively. After a few thousand of itera-

a)

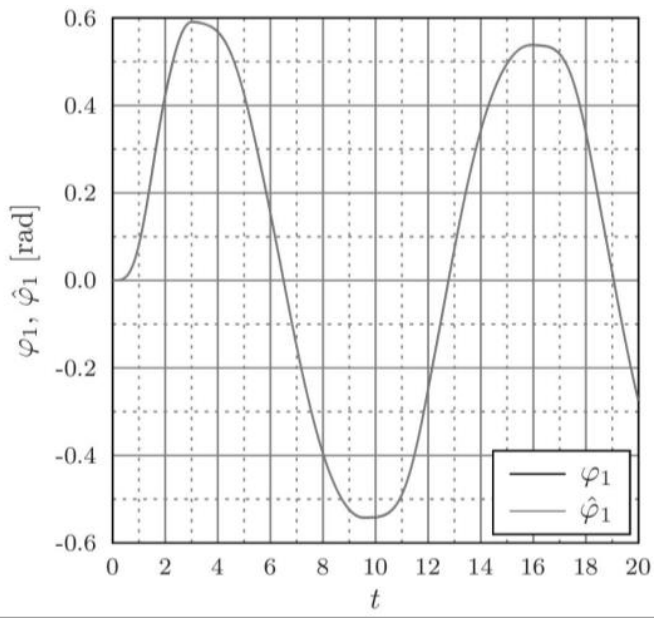

tions, the smallest possible value of objective function was obtained: $y=0.01171$. b)

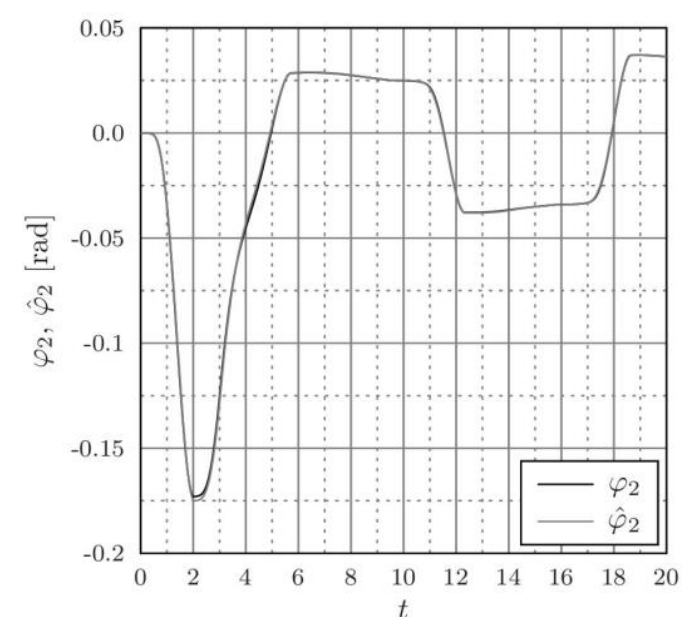

Fig. 11. Time histories of the actual $\left[\varphi_{1}, \varphi_{2}\right]$ and estimated $\left[\hat{\varphi}_{1}, \hat{\varphi}_{2}\right]$ dynamical variables of the pendulum after final identification of parameters. In the presented view, the model's parameters are identified (the procedure of identification is finished)

a)

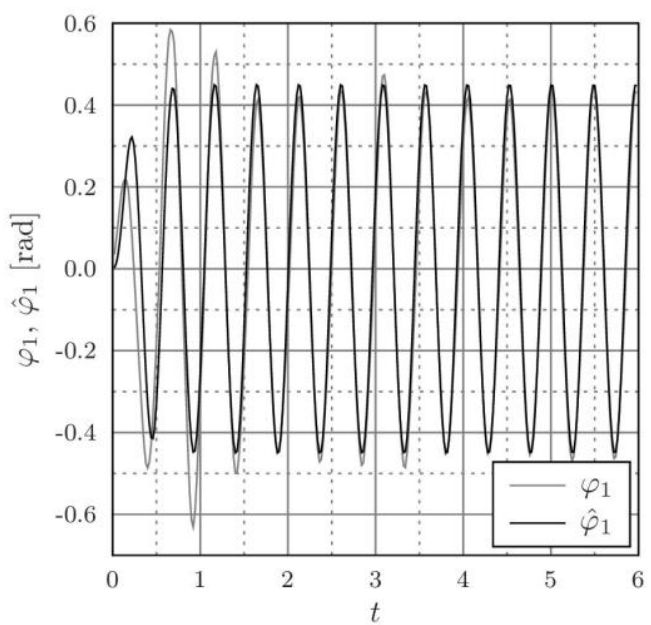

b)

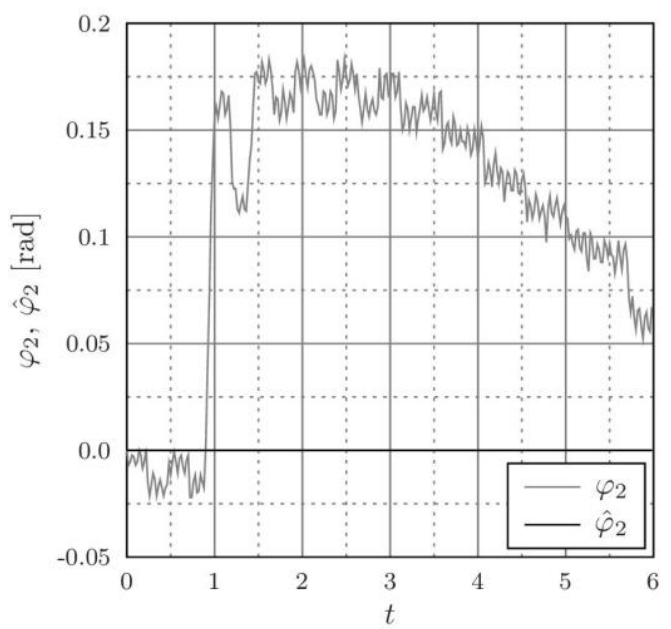

Fig. 12. Time histories of the actual $\left[\varphi_{1}, \varphi_{2}\right]$ and estimated $\left[\widehat{\varphi}_{1}, \widehat{\varphi}_{2}\right]$ dynamical variables of the pendulum after final identification of parameters

a)

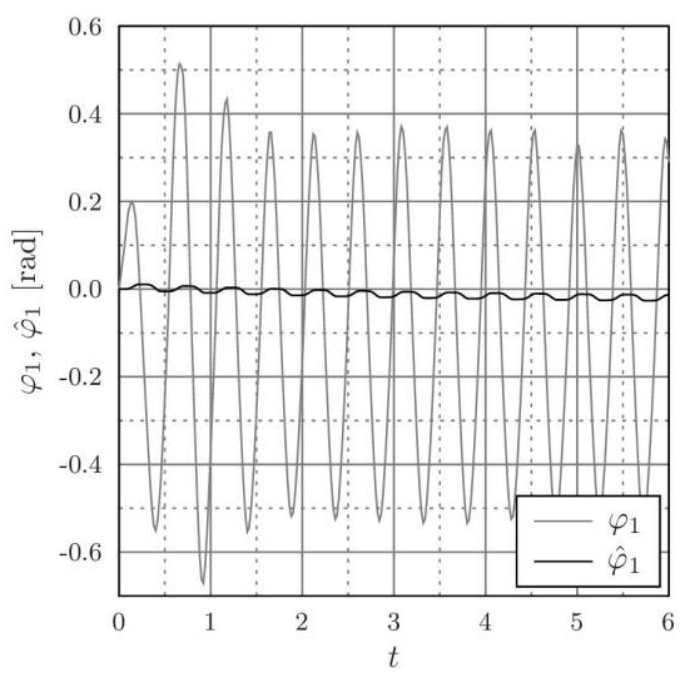

b)

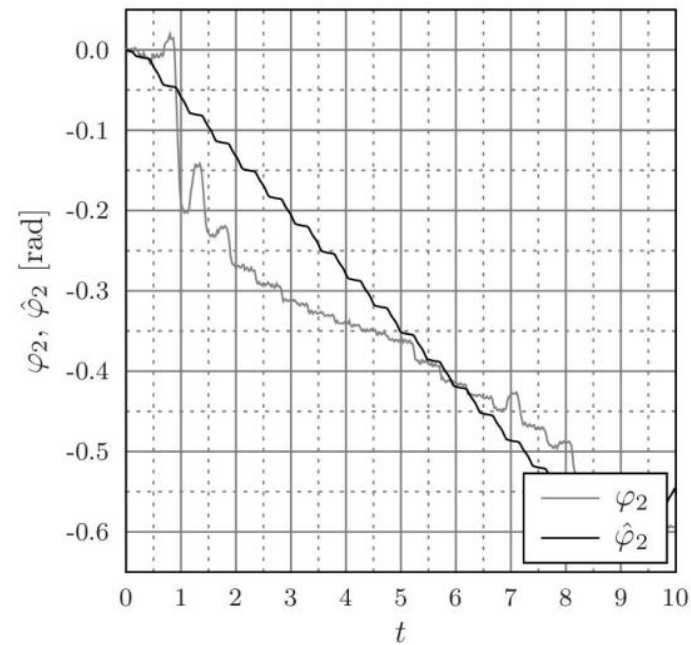

Fig. 13. Time histories of the actual $\left[\varphi_{1}, \varphi_{2}\right]$ and estimated $\left[\hat{\varphi}_{1}, \hat{\varphi}_{2}\right]$ dynamical variables of the analyzed pendulum after final identification of parameters 
Given the time history in Fig. 12b, which shows the matching, it can be concluded, that the presented numerical solution of the double torsion pendulum's model with friction does not match the measurement series obtained from our real experiment. The time history of actual value and the estimated value of the angle $\varphi_{1}$ tends to correct convergence, however, the $\varphi_{2}$ angle's time history does not show any acceptable convergence.

Then, the second real identification was performed. As previously, three parameters were assumed as known and set to the same values. After a few thousand of iterations, the smallest possible value of objective function was obtained: $y=0.05328$.

Given the time history in Fig. 13, which shows the matching, it can be concluded, that the presented numerical solution of the double torsion pendulum's model with friction does not satisfctorily match the measurement series of data obtained from our real experiment as in the previous case.

\section{CONCLUSIONS}

The introduced mathematical model of the torsion double pendulum with a spiral spring allows to simulate complex dynamics of the mechanical system. The time histories of angular displacement, velocity and acceleration of the pendulum's column were shown in Fig. 9. According to the given time history of external excitation of the pendulum, the kinematic forcing takes the form of sine wave. It is one of the reasons causing main complexity of the system, so the angular velocity and acceleration are difficult to predict. This irregularity of responses is caused by the effects of dry and viscous friction acting on the column and the free body placed on its head. In turn, we had shown in Fig. 10 a time history of the angular displacement, velocity and acceleration of the free body, that is forced via a plane frictional contact by the column's head. We can see the restraint of the free body against the column. Also in this case, if we consider a noise in the measured series of data, then the velocity and acceleration will be impossible to observe. After the first sample simulations, it was decided to carry out the identification of model parameters using a slightly modified form in relation to the simulation model. It has led to some satisfactory fitting, even though not all the parameters have been fully identified. This may be due to the fact that this particular solution was possible with several different values of unknown parameters. It is worth noting that even a small change in the parameters dramatically changes the behavior of our mechanical system.

The next step was to perform the identification of pendulum's parameters. Considering the real and the estimated angular displacements of the pendulum's bodies, as well as assuming that the parameters obtained with known parameters $B_{1}$ and $B_{2}$ should be equal to each other, it can be concluded, that the matching of the double torsion pendulum's model with a planar frictional contact to the measurements obtained from the real experiment is not sufficiently correct. There are several aspects that have a direct impact on such divergence.

Firstly, it should be pointed out that the system is very sensitive and has a lot of local solutions dependent on initial conditions. Free body of the torsion pendulum can move relatively to the column, where it can exhibit an oscillatory rotational motion to the right and then to the left, it can move only in one direction and suddenly stop to move with the column, it can sometimes exhibit rapid stick-slip behavior in relation to the pendulum's head and others. Even with the same initial conditions, the system may behave differently as shown by measurements. Another problem is devoted to the number of unknown parameters. To many unknown parameters were reported, so that the same co-existing solutions may be obtained at their different sets of values. It is also essential that the parameters of the model do not need to be constant in time, as it has been assumed at the beginning, and may vary in time.

The spiral spring is worth a comment. Its stiffness during winding and unwinding is slightly different, although it is assumed constant. Another imperfections can be introduced by small vibrations visible on the graphs of the angular displacement of the free body. Such discrepancy may be caused by insufficiently precise manufacturing of the mechanical parts of the laboratory stand, the type of bearing and insufficient parallelism between the base and the column, which causes vibration of the column in a vertical plane. Another problem could be deduced from the mathematical model, in which, there could not be taken into account the unknown dynamical effects that occur in the adequate real object, or even the idealized mathematical model could include too many frictional effects that complicate the identification. A small change of the parameters may result in a large change in the waveforms of the double torsion pendulum's states. In most considered cases, the estimated displacement of the column adjusts to a measured position as the parameters in the equation of the position of the columns are easier to estimate, and the second equation depends only on both the movement of the column and the friction between the column and the free body.

Another aspect may be connected with the Nelder-Mead method. Despite the fact that it is a simple and easy implementing method allowing the determination of extremes of nonlinear function of several variables without the use of derivatives, the objective function can reach local minima during the identification algorithm, so it is not always possible to find the correct set of identified parameters.

The last aspect that one needs to consider is the fact that the arm of the mechanism realizing the dynamic kinematic forcing is greater than the spring hook arm, which can cause unnatural deformation of the spring and the fact, that angle-voltage characteristics of two sensors are not ideal to measure the rotational movement of the column and the free body in a range greater than $\pm 45^{\circ}$. This prevents a full examination of the model, as in other cases. In the measurements, it was necessary to adopt high frequency of excitation, which leads to the observed rapid dynamic changes of state variables.

Despite the not fully correct identification of parameters of the double torsion pendulum with friction, we have achieved a good basis for further work on the subject. It is possible to consider several possible paths, such as simplifications based on reduction of system variables, or the extension of the model to take into account more effects, including a time-dependent uncertainty of model parameters, which were originally assumed as constants. One should also tend to improve accuracy of the angle sensors to be able to measure the states more correctly. Performance of certain mechanical parts should be raised by eliminating unwanted vibrations of the column or by replacing the bearing with another one of a higher enough tolerances, as well as by using a spiral spring with a greater stiffness. The identification method could be supplemented with partial analytical solutions to identify some unknown parameters. 


\section{REFERENCES}

1. Awrejcewicz J., Olejnik P. (2005), Analysis of dynamic systems with various friction laws, Applied Mechanics Reviews - Transactions of ASME, Vol. 58 (6), 389-411.

2. Awrejcewicz J., Olejnik P. (2005), Friction pair modeling by 2-dof system: numerical and experimental investigations, International Journal of Bifurcation and Chaos, Vol. 15 (6), 1931-1944.

3. Awrejcewicz J., Olejnik P. (2007), Occurrence of stick-slip phenomenon, Journal of Theoretical and Applied Mechanics, Vol. 45(1), 33-40.

4. Bassan M., De Marchi F., Marconi L., Pucacco G., Stanga R., Visco M. (2013), Torsion pendulum revisited, Physics Letters A, Vol. 377 (25-27), 1555-1562.

5. Cadoni M., De Leo R., Gaeta G. (2013), Solitons in a double pendulums chain model, and DNA roto-torsional dynamics, Journal of Nonlinear Mathematical Physics, Vol. 14(1), 128-146.

6. Coullet P., Gilli J.-M., Rousseaux G. (2009), On the critical equilibrium of the spiral spring pendulum, Proceedings of the Royal Society A, Vol. 466, 407-421.

7. De Marchi F., Pucacco G., Bassan M., De Rosa R., Di Fiore L., Garufi F., Grado A., Marconi L., Stanga R., Stolzi F., Visco M. (2013), A quasi-complete mechanical model for a double torsion pendulum, Physical Review D, Vol. 87(122006).

8. Liu X., Vlajic N., Long X., Meng G., Balachandran B. (2014), State-dependent delay influenced drill-string oscillations and stability analysis, ASME Journal of Vibration and Acoustics, Vol. 136(5), 051008.

9. Luersen M.A., Le Riche R. (2004), Globalized Nelder-Mead method for engineering optimization, Computers \& Structures, Vol. 82(23-26), 2251-2260.

10. Miao C., Luo W., Ma Y., Liu W., Xiao J. (2014), A simple method to improve a torsion pendulum for studying chaos, European Journal of Physics, Vol. 35, 055012.

11. Michalak M., Krucińska I. (2004), Studies of the effects of chemical treatment on bending and torsional rigidity of bast fibres, Materials Science, Vol. 10(2), 182-185.

12. Skup Z. (2002), Structural friction and viscous damping in a frictional torsion damper, Journal of Theoretical and Applied Mechanics, Vol. 2(40), 497-511.

The work has been supported by the Polish National Science Centre, MAESTRO 2, No. 2012/04/A/ST8/00738 and it was presented during the 3rd International Conference on MECHATRONICS - Ideas for Industrial Applications, Gdansk, 2015.

\section{APPENDIX}

Listing 1. A procedure in Scilab of using the Nelder-Mead identification method

ExpData = fscanfMat("data_in_rad.txt");

fip1.time $=\operatorname{ExpData}(:, 1)$;

fip1.values $=$ ExpData(:,2);

fip2.values $=\operatorname{ExpData}(:, 3)$;

function $\mathbf{y}=\mathrm{Fobj}(\mathbf{x})$;

importXcosDiagram ("xcos_model_pendulum.zcos")

Context. $w=13.09 ;$ Context.E1=1000; Context.E2=1000;

Context.B1=x(1);Context.B2=x(2); Context.k=x(3);

Context.A=x(4);Context.c1=x(5);Context.M=x(6);

Context.Tv=x(7);Context.Ts1=x(8);Context.Ts2=x(9);

Context.Tst=x(10);Context. T0=x(11);

Context.tmax $=10 ;$ Context.tini $=0 ;$ Context.S=2;

scicos_simulate(scs_m,Context);

$y=\left(\right.$ mean $\left((f i p 1 . v a l u e s-f i 1 . v a l u e s)^{\wedge} 2\right)+\underline{\text { mean }}(($ fip2.values-

fi2.values $\left.\left.)^{\wedge} 2\right)\right) / 2$;

clf(1); figure(1)

plot(fip1.time, [fip1.values, fi1.values]);

clf(2); figure(2)

plot(fip1.time, [fip2.values,fi2.values]);

endfunction

$x=[1,1,1,1,1,1,1,1,1,1,100]$

$y=$ Fobj $(x)$

opt = optimset("Maxlter",50,"PlotFcns",optimplotfval);

$x=\underline{\text { fminsearch }}$ (Fobj, $x, o p t)$ 